\title{
INDICADORES DE DESEMPEÑO AMBIENTAL EN LA EXTRACCIÓN DE RECURSOS NO RENOVABLES
}

\section{ENVIRONMENTAL PERFORMANCE INDICATORS IN THE EXTRACTION OF NON- RENOVATIVE RESOURCES}

Clara Luz Reynaldo Argüelles, Ph.D.

Doctora en Ciencias Económicas (Cuba). Directora del Centro de Estudios del Medio Ambiente (CEMA), Cuba. creynaldo1982@gmail.com

Roberto Guillermo Aguilera Peña, MsC. Máster en gestión y auditoría medioambiental (España). Docente a tiempo completo de la Facultad de Turismo y Hotelería de la Universidad Tecnológica ECOTEC, Ecuador. raguilera@ecotec.edu.ec

\section{ARTÍCULO DE INVESTIGACIÓN}

Recibido: 9 de septiembre de 2019.

Aceptado: 31 de octubre de 2019.

\section{RESUMEN}

La Evaluación del Desempeño Ambiental (EDA) es un proceso que facilita las decisiones de la dirección con respecto a la responsabilidad ambiental. Se sustenta en indicadores que proporcionan información oportuna, basada en la comparación entre el desempeño ambiental pasado y actual de la organización. El desarrollo económico internacional se ha caracterizado por una creciente comercialización de recursos no renovables, entre ellos, los minerales, encontrados debajo de la corteza superficial de la tierra. Los métodos utilizados para su extracción, originan externalidades ambientales, visuales, humanas y culturales. La intensidad de su impacto dependerá directamente del tipo de mineral que se pretenda extraer. La necesidad de contar con indicadores para medir el desempeño ambiental en la 
extracción de recursos no renovables surge por la preocupación de actuar con inmediatez ante los casi irreversibles efectos negativos que ocasiona esta actividad, de naturaleza no sustentable, pues supone el agotamiento del recurso en cuestión. El presente trabajo persigue como objetivo: proponer indicadores de desempeño ambiental para la extracción de recursos no renovables, a través de la medición de impactos ambientales y con la utilización de métodos estadístico-matemáticos. Los indicadores propuestos contribuirán con la utilización racional de los recursos naturales, la minimización de externalidades y la eficiencia económica empresarial.

Palabras clave: indicadores de desempeño ambiental, extracción de recursos no renovables.

\section{ABSTRACT}

The Environmental Performance Assessment (EDA) is a process that facilitates management's decisions regarding environmental responsibility. It is based on indicators that provide timely information, based on the comparison between past and current environmental performance of the organization. International economic development has been characterized by a growing commercialization of non-renewable resources, e.g. minerals, found beneath the earth's surface crust. The methods used originate environmental, visual, human and cultural externalities. The intensity of its impact will depend directly on the type of ore it is intended to extract. The need for indicators to measure environmental performance in the extraction of non-renewable resources arises from the concern to act immediately in the face of the almost irreversible negative effects of this activity, of a non-renewable nature it is the exhaustion of the resource in question. This work aims to: to propose environmental performance indicators for the extraction of nonrenewable resources, through the measurement of environmental impacts and with the use of statistical-mathematical methods. The proposed indicators will contribute to: the rational use of natural resources, the minimization of externalities and business economic efficiency.

Keywords: environmental performance indicators, non-renewal resource extraction. 


\section{INTRODUCCIÓN}

Con la definición de Desarrollo Sostenible presentada por la doctora Gro Harlem Brundtland, en el año 1987 en las Naciones Unidas, documento originalmente denominado Nuestro Futuro Común (Our Common Future, en inglés), comenzó a apreciarse en todos los sectores de la economía del escenario internacional, una concientización por la protección del medio ambiente. Este fenómeno ha sido impulsado principalmente por la legislación asociada, derivada de la necesidad de conservación de recursos naturales y reducción de impactos asociados al desempeño ambiental de las organizaciones.

En la búsqueda de modos de entender, demostrar y mejorar su desempeño ambiental, muchas empresas han entendido que este fin se logra con una gestión eficaz de aquellos elementos que pueden tener un impacto significativo en el medio ambiente, tal es el caso de la extracción de los recursos no renovables.

En este escenario, el tratamiento de la información ambiental y la obtención de un número limitado de indicadores claves se convierten en elementos críticos para el éxito de la organización, ayudando a los directivos en el proceso de toma de decisiones ambientales.

Según Henri \& Journeault (2008) la importancia de la medición se refiere a la atención dedicada por las empresas para cuantificar diversas cuestiones ambientales. Investigaciones previas han identificado cuatro dimensiones de desempeño ambiental que los indicadores ambientales deben medir: interna, externa, procesos y el resultado (Lober, 1996; Ilinitch et al., 1998).

Esta necesidad creciente de mejora continua del desempeño ambiental de las organizaciones y el incremento gradual de la legislación regulatoria asociada a la protección del medio ambiente, ha estimulado la investigación en este campo como, Luz et al. (2006), Ramos \& Melo (2006), Gunningham (2009), Sellitto et al. (2010) y Raupp (2012). 
En los últimos treinta años el pensamiento y la acción ambiental internacional ha crecido y se ha perfeccionado, en el aspecto legislativo, político, educativo, científico, tecnológico, social y económico; influyendo positivamente en el proceso de toma de decisiones. Sin embargo, aún existen deficiencias en relación con la evaluación del desempeño ambiental, como proceso de gestión interna de las organizaciones, que ayude a seleccionar, recopilar, integrar y evaluar indicadores ambientales. Importantes aportes se evidencian en las investigaciones de Parra (2005), Broche \& Ramos (2010), Isaac et al. (2010) y Sagastume et al. (2012); pero aún no se identifica un método de desempeño organizacional basado en indicadores operacionales y de gestión, que brinden información condensada del desempeño respecto a las metas organizacionales, facilitando la identificación de puntos críticos y oportunidades de mejoras.

El objetivo general del artículo, es presentar contribuciones teóricas para la evaluación del desempeño ambiental, a través del diseño y propuesta de indicadores ambientales y su evaluación haciendo uso del índice global de desempeño ambiental (IGDA); que facilite la identificación de puntos críticos y oportunidades de mejora.

El tema de la extracción de recursos no renovables, dentro de ellos, los minerales, constituye un tema de importante atención en agendas nacionales e internacionales por el impacto económico que genera en las economías y la relación desproporcional con el compromiso ambiental contraído por los actores que lo desarrollan. Para la aplicación de los indicadores se escogió el procesamiento del mineral cobalto en una comunidad de Cuba.

Luego de la introducción, el artículo presenta: (i) revisión teórica sobre la evaluación del desempeño e indicadores ambientales, índices agregados y métodos multi-criterio; (ii) metodología de la pesquisa; (iii) resultados de la aplicación del procedimiento propuesto; (iv) conclusiones y trabajos futuros.

\section{REVISIÓN TEÓRICA}

1.1. Evaluación del desempeño ambiental e indicadores ambientales. 
El desempeño ambiental es considerado un concepto derivado de la gestión ambiental, son los resultados medibles de la gestión que hace una organización de sus actividades, productos y/o servicios que puede interactuar con el medio ambiente. Las organizaciones no solo deben conocer su desempeño ambiental sino evaluarlo para identificar avances y retrocesos en la relación de la organización con el medio ambiente. Por su parte, la evaluación del desempeño ambiental (EDA) está basada en la máxima, "lo que no puede ser medido, no puede ser gestionado".

Según la Norma ISO 14001: 2015, la EDA es el proceso utilizado para facilitar las decisiones de la dirección con respecto al desempeño ambiental de la organización mediante la selección de indicadores, la recopilación y el análisis de datos, la evaluación de la información comparada con los criterios de desempeño ambiental, los informes y comunicaciones, las revisiones periódicas y las mejoras de este proceso.

Isaac-Godínez et al. (2010) plantea que la evaluación del desempeño debe estar relacionada con las características de la organización y de sus procesos sustantivos, ser capaz de estructurar y proporcionar la información para la toma de decisiones, y la comunicación de una gestión eficaz en función del cuidado del medio ambiente. La evaluación del desempeño ambiental debe ser multidimensional y no limitarse a informes, pues estos no integran los múltiples factores que afectan el desempeño ambiental empresarial.

Más contextualizado con el escenario actual, la Norma Internacional ISO 14001 del año 2015 relacionada con el Sistema de Gestión Ambiental (SGA), define a la evaluación del desempeño ambiental como el resultado medible del SGA, relacionado con el control de los aspectos ambientales de una organización, con base en su política, objetivos y metas. Se observa claramente una distinción fundamental basada en el alcance del concepto, ya que la definición de ISO 14001 no se limita a los resultados provenientes del Sistema de Gestión Ambiental implementado, sino a la gestión ambiental de una organización, tenga o no, un SGA. 
En el capítulo 9, la Norma ISO 14001:2015 establece que la organización debe hacer seguimiento, medir, analizar y evaluar su Desempeño Ambiental.

Concretamente, esta norma define que:

La organización debe determinar:

a) qué necesita seguimiento y medición;

b) los métodos de seguimiento, medición, análisis y evaluación, según corresponda, para asegurar resultados válidos;

c) los criterios contra los cuales la organización evaluará su desempeño ambiental, y los indicadores apropiados;

d) cuándo se deben llevar a cabo el seguimiento y la medición;

e) cuándo se deben analizar y evaluar los resultados del seguimiento y la medición.

La Evaluación del Desempeño Ambiental (EDA) es un proceso utilizado para facilitar las decisiones de la dirección con respecto al desempeño ambiental de la organización, y que utiliza indicadores para proporcionar información, comparando el desempeño ambiental pasado y actual de una organización. La EDA sigue el modelo de "Planificar-Hacer-VerificarActuar".

A su vez, el Desempeño Ambiental (DA) se define como el conjunto de resultados medibles de la gestión que hace una organización de sus aspectos ambientales.

\subsection{Elementos de la Evaluación del Desempeño Ambiental (EDA).}

La Evaluación del Desempeño Ambiental debe ser apropiada para las características de la organización o empresa, y debe resultar rentable y hacer parte de las actividades habituales de la organización. Los elementos claves que componen la Evaluación del Desempeño Ambiental son los siguientes:

- Selección de indicadores 
- Recopilación y análisis de datos

- Evaluación de información comparada con los criterios de desempeño ambiental

- Elaboración de informes y comunicaciones

- Revisiones periódicas y mejoras de este proceso

\subsection{Beneficios de la Evaluación del Desempeño Ambiental.}

Entre los beneficios de la EDA se pueden mencionar los siguientes:

- Mejora la comprensión de los impactos ambientales de la organización

- Provee la base para el desempeño operacional, ambiental y de "benchmarking"

- Identifica oportunidades para mejorar la gestión de sus aspectos ambientales

- Verifica cumplimiento de objetivos y requisitos legales

- Determina la utilización correcta de recursos

- Aumenta la conciencia de los empleados

- Determina acciones necesarias para el logro de los criterios del desempeño ambiental

- Identifica aspectos ambientales importantes y tendencias en el desempeño ambiental

- Incrementa la eficiencia y la eficacia de la organización

- Mejora las relaciones con la comunidad y otras partes interesadas

\subsection{Los indicadores de desempeño ambiental.}

Los indicadores de desempeño ambiental son la base de la EDA y miden el desempeño ambiental actual o pasado de una organizacion y lo compara contra las metas definidas por la dirección (Ormazabal \& Larrañaga, 1999; Jasch, 2000; Herva et al., 2011), representan la cuantificación de la efectividad y eficiencia de las acciones ambientales con un conjunto de métricas (Neely et al., 1995) y hacen referencia a la medida de interacción entre la organización y el medio ambiente (Olsthoorn et al., 2001). También representan las 
mediciones cualitativas y cuantitativas, financieras o no financieras, que proporcionan información importante sobre el impacto ambiental, cumplimiento regulatorio, las relaciones con las stakeholders y los sistemas organizacionales (Henri \& Journeault, 2008).

Las estructuras analíticas de los sistemas de indicadores ambientales más usadas, son las siguientes:

1. Indicadores según modelo de Presión-Estado- Respuesta (PER), introducido por la Organización para la Cooperación Económica y Desarrollo 1994 (OECD, siglas en inglés).

2. Indicadores de desempeño ambiental y de condición ambiental Norma ISO 14031-2005.

3. Indicadores para comunicar el desempeño ambiental según Global Reporting Initiative (GRI, 2006).

\subsection{Los recursos no renovables}

Dentro de los recursos naturales de que dispone el hombre en el planeta, existen aquellos que pueden renovarse por medios naturales como los recursos pesqueros o los bosques, su rehabilitación por parte de los mecanismos naturales es lenta y puede tardar años, décadas e incluso más, pero se regeneran. A estos recursos se les conoce como recursos renovables.

Existe otro tipo de recursos que se encuentran en la naturaleza y no se regeneran, son explotados por el ser humano y cuya disponibilidad en la naturaleza disminuye sin que exista un mecanismo natural que permita su reposición. A estos se les denomina recursos no renovables.

Los recursos no renovables son limitados y se agotarán con el tiempo. Los minerales constituyen recursos no renovables y se pueden citar como ejemplos: aluminio, carbón, cobalto, cobre, cromo, diamante, esmeralda, estaño, hierro, magnesio, mercurio, níquel, oro, petróleo, plata, platino, plomo, rubí, zinc, entre otros. Ver Figura 1 


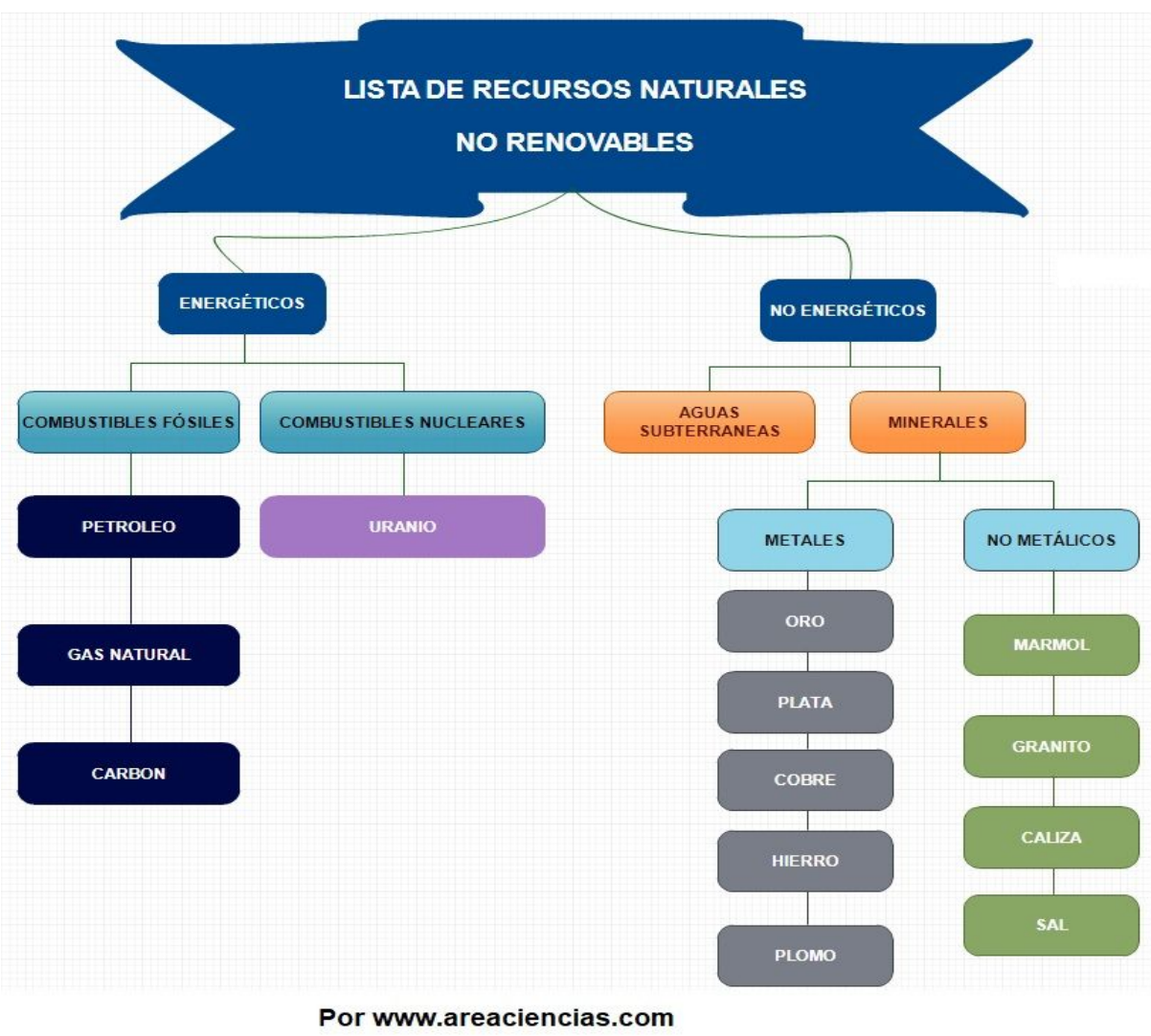

Figura 1. Recursos Naturales no renovables.

Fuente: Elaboración propia.

El consumo excesivo de recursos no renovables ha ocasionado que éstos disminuyan de forma considerable, lo que genera acciones necesarias como la restricción de su explotación y del uso desmedido, así como el reciclaje de aquellos materiales que se puedan reutilizar. Sin embargo, el ritmo actual de extracción de recursos no renovables, contribuirá, en un futuro cercano, a su agotamiento.

\subsection{La extracción de minerales y su expresión en la economía de los países.}

Los minerales se consideran recursos no renovables que no pueden ser producidos, regenerados o reutilizados a una escala tal que pueda sostener su tasa de consumo. Estos recursos frecuentemente existen en cantidades fijas o son consumidos mucho más rápido 
de lo que la naturaleza puede recrearlos, por lo que al referirse a su localización se utiliza el término reservas.

Se llama reservas a los contingentes de recursos que pueden ser extraídos con provecho. El valor económico (monetario) depende de su escasez y demanda, y es un tema que preocupa a la economía. Su utilidad como recursos depende de su aplicabilidad, pero también del costo económico y del costo energético de su localización y explotación. Por ejemplo, si para extraer un mineral hay que invertir más energía que la que va a proporcionar, no puede considerarse un recurso. Es por ello que uno de los recursos no renovables son los minerales.

La contabilidad de las reservas produce muchas disputas, con las estimaciones más optimistas por parte de las empresas, y las más pesimistas por parte de los grupos ecologistas y los científicos académicos. Donde la confrontación es más visible es en el campo de las reservas de hidrocarburos; aquí, los primeros tienden a presentar como reservas todos los yacimientos conocidos más los que prevén encontrar. Los segundos ponen el énfasis en el costo monetario creciente de la exploración y de la extracción, y en el costo termodinámico (energético) creciente, que disminuye el valor de uso medio de los nuevos hallazgos.

La minería puede brindar importantes beneficios económicos. Esto reviste especial importancia para aquellos países y regiones pobres carentes de otras fuentes de desarrollo y que, en otras circunstancias, no serían atractivos para la inversión. Es probable que, cumplidas ciertas condiciones -como un marco legal y político adecuado, un nivel aceptable de estabilidad política y derechos de propiedad bien definidos-, los inversionistas extranjeros se sientan atraídos hacia depósitos ricos en minerales.

Aunque muchos países han obtenido grandes beneficios de la extracción de minerales, por distintas razones, otras naciones no han logrado capitalizar las oportunidades dadas por la minería. La capacidad para administrar la riqueza mineral de manera eficaz ha sido más lenta que la capacidad para atraer la inversión. Un desafío clave para muchos países ahora es elaborar marcos políticos para asegurar la captación de la riqueza mineral y la 
generación de beneficios duraderos para las comunidades locales y el grueso de la población. Este marco debe reconocer que la producción de un yacimiento determinado tiene una duración finita; cuando la mina cierre, es fundamental que quede algo para mostrar en valores mejorados $u$ otra forma de capital.

Un desafío mayor para los países productores consiste en ser capaces de aumentar el valor agregado de los minerales. En especial, se deben dar mayores oportunidades a los países en desarrollo para que logren esto. Los mercados que reciben productos primarios no tienen por qué discriminar los productos que han sido sometidos a un mayor procesamiento en el país exportador.

\subsection{Necesidad de medir el desempeño ambiental en la extracción de minerales como recursos no renovables.}

Los indicadores de desempeño ambiental en la extracción de minerales como recursos no renovables, son parte del Sistema de Información Ambiental que proporcionará información al comparar el desempeño ambiental pasado y presente, con respecto a los objetivos o metas ambientales propuestos para el Sistema de Gestión Ambiental. La metodología desarrollada para el diseño de los indicadores debe ser coherente con los objetivos y metas ambientales del Sistema de Gestión Ambiental en el ciclo Planificar-Hacer-Verificar-Actuar "PHVA" (ISO 14001) utilizando el modelo de la norma ISO: "Evaluación del desempeño ambiental", cuya metodología se basa en el mismo ciclo por ser parte del compendio de normas ISO 14000. El diseño de indicadores ambientales organizados como sistema, contribuirá con la medición de la gestión en los aspectos ambientales en la extracción de minerales, de acuerdo con los propósitos de las herramientas de gestión y las necesidades ambientales de la organización. 


\section{MATERIALES Y MÉTODOS}

\section{1. Índices agregados}

Existe gran tendencia a la construcción de índices agregados en el área ambiental y de la sostenibilidad. Las stakeholders prefieren los índices y además le permite a la empresa no dar información detallada del funcionamiento en sí del sistema, pero si dan una idea de su desempeño. Ejemplos de la literatura consultada, por solo citar algunos, lo demuestran Puolamaa et al. (1996), Chiang \& Lai (2002), Ramos \& Melo (2006), Singh et al. (2007), Blanc et al. (2008), Hubbard (2009), Sellitto et al. (2010) y Broche \& Ramos (2010).

Para la construcción de índices agregados de cualquier índole es necesario una serie de pasos entre los que se encuentran: la selección de los indicadores, la homogenización, la normalización, la ponderación y la agregación.

- Selección: proceso de decisión de los indicadores que integrarán el índice agregado.

- Homogenización: es el paso de llevar los indicadores seleccionados de naturaleza diferente a un mismo criterio ya sea de maximizar o minimizar.

- Normalización: los indicadores que componen los índices están distribuidos sobre diferentes categorías por lo que es necesaria una unidad común o equivalente. Algunos de los métodos más usados en la literatura para la normalización son: Z-score, normalización lineal, Min. -Máx. y otros como la normalización difusa.

- Ponderación: proceso para determinar y asignar las importancias relativas de los indicadores basado en criterio de expertos. Según Paoli \& Moraes (2011), los métodos multi-criterio están siendo utilizados en evaluaciones de impactos ambientales. Una de las técnicas más usadas para ponderar índices ha sido AHP (Analytic Hierarchy Process).

- Agregación: es el resumen de la información en un valor único, logrando el índice deseado. 
Según Diewert (1976) varias reglas y formas funcionales para la agregación de índices compuestos se han desarrollado en la literatura. Algunos de los métodos más usados son: el método de la utilidad aditiva, producto ponderado, media geométrica ponderada.

\subsection{Métodos multi-criterio}

AHP es una técnica de decisión multi-criterio, la cual fue desarrollada por Saaty (1980). Según Hernández et al. (2010) el $A H P$ es el método de decisión multi-criterio más referenciado en la literatura en los últimos 20 años. Otros como Hermans et al. (2008) plantean que esta ha sido una de las técnicas más usadas para la evaluación de los pesos de los indicadores ambientales tomando como ejemplos: Indoor Environment Index (Chiang \& Lai, 2002) y Environmental Friendliness (Puolamaa et al., 1996). También Saaty (2003) plantea que los pesos de los indicadores de sostenibilidad son generalmente obtenidos haciendo uso del método de decisión AHP.

En los últimos años varias investigaciones han ilustrado la preferencia de asignación sobre ciertos atributos por encima o por debajo de otros siempre que la información no esté completa. El campo de la ingeniería ambiental y la sostenibilidad no han escapado a esta preferencia, algunos ejemplos de la aplicación del AHP en estas áreas se pueden apreciar en Luz et al. (2006), Singh et al. (2007) y Hernández et al. (2010).

A pesar de la amplia aceptación del AHP en la construcción de índices, este brinda una visión poco realista de fenómenos naturales ya que parte de la independencia que debe de existir entre los niveles y alternativas del modelo. En ocasiones estas relaciones suelen ser más complejas, con un mayor número de relaciones e interrelaciones convirtiendo el modelo jerárquico en una estructura compleja.

Saaty desarrolló, el método ANP (Analytic Network Process). ANP provee una herramienta para lidiar con las decisiones sin asumir la independencia de los elementos de un nivel superior a los elementos de un nivel inferior y sobre la independencia de los elementos dentro de un nivel en una jerarquía. El ANP hace una extensión del método AHP para los 
problemas con dependencias y retroalimentación entre los criterios usando el enfoque de la "súper-matriz" (Saaty, 1996). Según Hernández (2010), ANP no obedece al axioma de independencia entre criterios o influencia entre alternativas.

Con respecto a la estructura de la decisión, Saaty \& Saaty (2003) observa que el ANP hace uso de las redes sin la necesidad de especificar niveles. Como en el AHP, la dominación o la importancia relativa de influencia es un concepto central, la ampliamente divulgada teoría multi-criterio AHP es un caso especial del ANP.

El ANP está compuesto por dos partes: 1) control de la jerarquía o la red de objetivos y criterios que controlan las interacciones del sistema bajo estudio y 2) muchas subredes de influencias entre todos los elementos y grupos del problema, uno por cada criterio de control.

Una red puede ser generada a partir de una jerarquía incrementando gradualmente las interconexiones, logrando las conexiones de los grupos como se deseen. Algunos grupos tienen elementos que se relacionan entre ellos lo que hace que presenten dependencia interna.

En los últimos años un aumento de la aplicación del ANP se evidencia en la producción científica; algunos ejemplos se pueden apreciar en Wang et al. (2006), Hernández et al. (2010), Hsu et al. (2011) y Lee et al. (2011), todos estos en diferentes áreas del conocimiento lo que da idea del éxito que ha tenido esta herramienta a nivel mundial.

\section{RESULTADOS}

Para evaluar el desempeño ambiental en la extracción de recursos no renovables como caso de estudio la extracción de cobalto en una comunidad de Cuba. Como período referencial se tomó el quinquenio 2010-2015 y se evaluaron los indicadores: producción 
física $(\mathrm{t})$, ventas netas (M USD), costos totales de producción $(\$ / \mathrm{lb}$ de $\mathrm{Ni}+\mathrm{Co})$, producción por procesos (t) y la incidencia de los costos de cada proceso en el costo de producción.

Para la identificación de los impactos ambientales se localizaron los expertos capaces de ofrecer valoraciones conclusivas de la temática, aplicando el método Delphi y calculando su Coeficiente de Conocimiento $\left(\mathrm{K}_{\mathrm{c}}\right)$, Coeficiente de Argumentación $\left(\mathrm{K}_{\mathrm{a}}\right)$ y Coeficiente de Competencia $\left(\mathrm{K}_{\mathrm{cm}}\right)$.

La Figura 2 muestra un diagrama con la secuencia lógica de la metodología propuesta para llegar al diseño de los indicadores de desempeño ambiental en la industria del cobalto.

Figura 2. Metodología propuesta para el diseño de los indicadores de desempeño ambiental en la industria del cobalto.

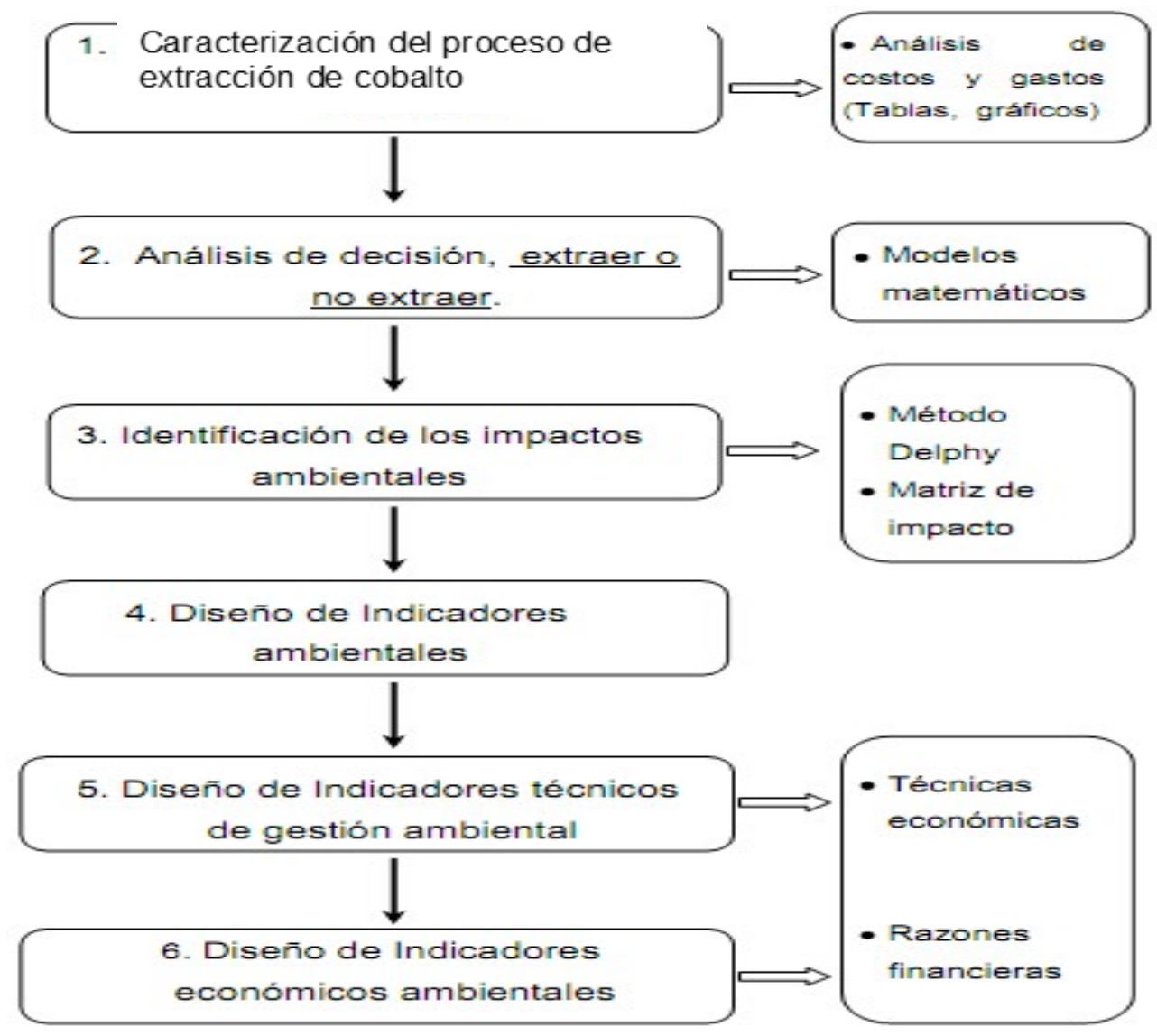

Fuente: Elaboración propia. 


\subsection{Caracterización del proceso de extracción de cobalto.}

En el período 2010-2015 la incidencia de los costos por procesos en el costo total de producción de cobalto en una de las comunidades mineras de Cuba, la posicionó desfavorablemente en la economía nacional con respecto a sus indicadores de eficiencia económica.

En el análisis detallado al informe técnico-económico se comprobó que dentro de los elementos de gastos solo se contemplan dos indicadores globales relacionados con la eficiencia del proceso de extracción: costo unitario de masa minera y costo unitario por cada libra de cobalto minado. Sin embargo, estos índices no reflejan realmente las causas de la incidencia del costo total de la actividad minera en el costo total de la empresa.

\subsection{Análisis de decisión}

La explotación de recursos no renovables lleva implícita dos decisiones: una de carácter empresarial y otra de carácter público. La aplicación de métodos y modelos económicos matemáticos permite calcular el nivel de ocurrencia y estimación en el comportamiento de las variables y la incidencia de unas con relación a las otras (De Castro, 2008).

La empresa puede decidir extraer o no extraer con la aplicación de las ecuaciones siguientes:

$$
\begin{aligned}
& p(t)[1+r(t)]>p(t+1) \Rightarrow \text { extraer } \\
& p(t)[1+r(t)]<p(t+1) \Rightarrow \text { no extraer } \\
& p(t)[1+r(t)]=p(t+1) \Rightarrow \text { indiferente }
\end{aligned}
$$

Donde:

(p) Precios

(r) Tipos de interés de mercado

(t) en función del tiempo

- La empresa puede decidir la cantidad a extraer 
El stock inicial es $\mathrm{x} 0$

En Tc se agota el recurso

$$
\begin{aligned}
& q^{c}(t)=d\left[p^{c}(t)\right] \\
& p^{c}(t)=p^{c}(0) e^{r t}=p^{c}\left(T_{c}\right) e^{-r T_{c}} e^{r t}=p^{m} e^{r\left(t-T_{c}\right)} \\
& q^{c}(t)=d\left[p^{m} e^{r\left(t-T_{c}\right)}\right] \\
& t=T_{c} \Rightarrow x_{0}=\int_{0}^{T_{c}} q^{c}(t) d t=\int_{0}^{T_{c}} d\left[p^{m} e^{r\left(t-T_{c}\right)}\right] d t
\end{aligned}
$$

La modelación matemática arrojó como resultado la decisión de extraer bajo condiciones de agotamiento acelerado de los recursos naturales, para lo cual se procedió a identificar los impactos ambientales en cada fase del desarrollo minero con la intención de hacer una utilización adecuada del escenario productivo.

\subsection{Identificación de los impactos ambientales durante el proceso de extracción de cobalto.}

De un total de 42 especialistas investigados, 14 tuvieron un coeficiente de conocimiento igual a uno $\left(\mathrm{K}_{\mathrm{c}}=1\right)$ y 16 resultaron entre 0,8 y 0,9 . Al evaluar el coeficiente de argumentación $\left(\mathrm{K}_{\mathrm{a}}\right)$ se obtuvo que 12 de ellos no poseían conocimientos técnicos sobre la temática, puesto que sus trabajos de investigación, experiencia y análisis teórico lo habían realizado en otro perfil. De forma general, 30 de los especialistas encuestados fueron evaluados como expertos con un coeficiente de competencia alto sobre el proceso de extracción de recursos no renovables y sus impactos ambientales. A los 30 especialistas revelados finalmente como expertos por su alto $\mathrm{K}_{\mathrm{cm}}$ se les aplicó la encuesta para la definición de los impactos ambientales de la actividad.

El procesamiento estadístico de las encuestas arrojó como resultado la identificación de 25 impactos ambientales. El criterio de los expertos reflejado en las encuestas permitió relacionar cada impacto ambiental con su nivel de incidencia. El procesamiento estadístico de las encuestas arrojó como resultado un listado de 25 impactos ambientales. 
El criterio de los expertos reflejado en las encuestas permitió relacionar cada impacto ambiental con la fase del proceso de extracción de cobalto de acuerdo con su nivel de incidencia. El análisis de la matriz dio como resultado que las actividades que mayor impacto ocasionan al medio ambiente son el desbroce y el destape del yacimiento.

\subsection{Diseño de indicadores de desempeño ambiental en la extracción de cobalto.}

El conjunto de indicadores de desempeño ambiental que se propone en la presente investigación incluye: indicadores ambientales, indicadores técnicos de gestión ambiental e indicadores económico-ambientales.

\subsubsection{Diseño de indicadores ambientales}

El consumo y utilización de los factores ambientales en la extracción de cobalto constituye un impacto directo del proceso productivo. La aplicación de métodos físicos permite identificar los indicadores ambientales, de acuerdo con la afectación de determinados factores ambientales (Tabla 1).

Tabla 1. Indicadores ambientales para la extracción de cobalto.

\begin{tabular}{|l|l|l|}
\hline Factor ambiental & $\begin{array}{l}\text { Indicador } \\
\text { Energía }\end{array}$ & $\begin{array}{l}\text { Consumo total de energía } \\
\text { Cantidad de electricidad consumida, } \\
\text { según fuente de combustible primaria }\end{array}$ \\
\hline Recursos energéticos & $\begin{array}{l}\text { - Consumo total de materias primas } \\
\text { (además de combustible y agua) }\end{array}$ \\
\hline $\begin{array}{l}\text { Agua } \\
\text { Emisiones, vertidos, } \\
\text { residuos y escombros }\end{array}$ & $\begin{array}{l}\text { - Consumo total de agua } \\
\text { Residuos (polvo, grasas) }\end{array}$ & $\mathrm{m3}$ \\
\hline
\end{tabular}


Utilización del suelo / biodiversidad
Tipo de hábitat y ecosistema afectado $\mathrm{m} 2$ Cambios en el hábitat debido a las operaciones de la organización

Superficie de hábitat protegido o restaurado Impacto sobre áreas protegidas (parques nacionales, reservas biológicas, zonas declaradas patrimonio universal...)

Fuente: Elaboración propia.

\subsubsection{Diseño de indicadores técnicos de gestión ambiental}

La aplicación de razones económicas posibilita relacionar el consumo de factores ambientales por cantidad de mineral extraído con las pérdidas ambientales, las materias primas, la energía y los residuos industriales (Tabla 2).

Tabla 2. Indicadores técnicos de gestión ambiental para la extracción de cobalto

\begin{tabular}{|l|l|l|}
\hline $\begin{array}{l}\text { Aspecto } \\
\text { ambiental }\end{array}$ & $\begin{array}{l}\text { Impacto } \\
\text { ambiental }\end{array}$ & Indicador de gestión \\
\hline $\begin{array}{l}\text { Consumo y } \\
\text { aguastaminación de }\end{array}$ & $\begin{array}{l}\text { Consumo de } \\
\text { aguas }\end{array}$ & $\begin{array}{l}\text { RC = Consumo de aguas / minerales } \\
\text { extraídos }\end{array}$ \\
\cline { 2 - 3 } & $\begin{array}{l}\text { Contaminación de } \\
\text { aguas }\end{array}$ & $\begin{array}{l}\text { REC = Miligramos de sustancias } \\
\text { contaminantes / m3 de agua }\end{array}$ \\
\hline $\begin{array}{l}\text { Consumo } \\
\text { energético y de }\end{array}$ & $\begin{array}{l}\text { Consumo de } \\
\text { materias primas }\end{array}$ & $\begin{array}{l}\text { RCMP = Consumo de Materias Primas / } \\
\text { minerales extraídos } \\
\text { RGR = Materiales no renovables / } \\
\text { materiales renovables }\end{array}$ \\
\cline { 2 - 3 } & Consumo & $\begin{array}{l}\text { RCE = Energías no renovables / } \\
\text { energías renovables }\end{array}$ \\
\hline $\begin{array}{l}\text { Generación y } \\
\text { tratamiento de } \\
\text { escombros }\end{array}$ & \begin{tabular}{l} 
REG = Escombros generados / minerales extraídos \\
\hline
\end{tabular} &
\end{tabular}


Donde:

RC: Razón de consumo de agua

REC: Razón de elementos contaminantes

RCMP: Razón consumo de materias primas

RGR: Razón de consumo de materiales renovables y/o no renovables

RCE: Razón de consumo de energías

REG: Razón de escombros generados

\subsubsection{Diseño de indicadores económico-ambientales}

Considerando la información que ofrecen los indicadores ambientales a partir de las unidades físicas consumidas por los factores ambientales en el proceso de extracción de cobalto y la posibilidad de valorar, en razones económicas, la cantidad de agua, materias primas, energía y escombro de acuerdo con la cantidad de mineral extraído, se diseñaron los indicadores económico-ambientales que incluyen criterios como: costos, gastos, ingresos, impuestos, inversiones y rentabilidad. (Tabla 3).

Tabla 3. Indicadores económico-ambientales

\begin{tabular}{|l|l|l|}
\hline \multicolumn{2}{|l|}{ Responsabilidad Ambiental } & RRA = Costos ambientales / Costos totales \\
\hline \multicolumn{2}{|l|}{ Inversiones Ambientales } & $\begin{array}{l}\text { RITL= Inversión en tecnologías } \\
\text { limpias/Activos totales } \\
\text { RAAM = Costos amortización de activos } \\
\text { ambientales / Costos amortización total }\end{array}$ \\
\hline \multirow{2}{*}{$\begin{array}{l}\text { Tratamiento de } \\
\text { residuos y consumos } \\
\text { energéticos }\end{array}$} & $\begin{array}{l}\text { Tratamiento } \\
\text { de }\end{array}$ & $\begin{array}{l}\text { RCTE = Costos transporte escombros / Costos } \\
\text { gestión escombros }\end{array}$ \\
\cline { 2 - 3 } & $\begin{array}{l}\text { Consumo } \\
\text { energético }\end{array}$ & $\begin{array}{l}\text { RCE = Consumo de energía / Costo industrial } \\
\text { RCRNR = Consumo de recursos no renovables }\end{array}$ \\
\hline
\end{tabular}




\begin{tabular}{|l|l|l|}
\hline \multicolumn{2}{|l|}{} & / Consumo total de recursos \\
\hline \multicolumn{2}{|l|}{ Riesgos Ambientales } & $\begin{array}{l}\text { RIA = Impuestos Ambientales / Impuestos } \\
\text { totales }\end{array}$ \\
\hline $\begin{array}{l}\text { Rentabilidad } \\
\text { Ambiental }\end{array}$ & $\begin{array}{l}\text { Ingresos y } \\
\text { gastos }\end{array}$ & RCA = Costos Ambientales/Costos totales \\
\cline { 2 - 3 } & Rentabilidad & $\begin{array}{l}\text { RGA=Ingresos Ambientales / Beneficios } \\
\text { Ambientales }\end{array}$ \\
\hline
\end{tabular}

Fuente: Elaboración propia.

Donde:

RRA: Razón de responsabilidad ambiental

RITL: Razón de inversiones en tecnologías limpias

RAAM: Razón de amortización de activos ambientales

RCTE: Razón del costo de transporte de escombros

RCE: Razón de consumo de energía

RCRNR: Razón de consumo de recursos renovables y no renovables

RIA: Razón de impuestos ambientales

RCA: Razón de costos ambientales

RGA: Razón de ingresos ambientales

\section{CONCLUSIONES}

La Economía Ambiental ofrece las herramientas económicas necesarias para lograr la utilización adecuada de recursos renovables y no renovables y contribuir con la eficiencia económica en la gestión ambiental de las organizaciones. 
Los indicadores ambientales son herramientas que no solo suministran información, también permiten el diagnóstico de aspectos ambientales, identificando alternativas y prioridades, además de los aspectos críticos que evalúan los esfuerzos de la organización por cumplir con las obligaciones ambientales consagradas en la política ambiental.

Los indicadores propuestos para evaluar el desempeño ambiental en la extracción de recursos no renovables constituyen una herramienta metodológica que facilita a administrativos y trabajadores de la minería contar con un sistema de indicadores que puede contribuir con la incorporación de la dimensión ambiental a la eficiencia empresarial y con la definición de prioridades en las decisiones de inversión.

\section{REFERENCIAS BIBLIOGRÁFICAS}

Blanc, I., Friot, D., Margni, M., \& Jolliet, O. (2008). Towards a new index for environmental sustainability based on a DALY weighting approach. Sustainable Development, 16(4), 251-260. http://dx.doi.org/10.1002/sd.376.

Broche, Y., \& Ramos, R. (2010). Procedimiento para la gestión de residuos generados en instalaciones hoteleras cubanas. Retos Turísticos, 9(3), 13-19.

Chiang, C. M., \& Lai, C. M. (2002). A study on the comprehensive indicator of indoor environment assessment for occupants' health in Taiwan. Building and Environment, 37(4), 387-392. http://dx.doi.org/10.1016/ S0360-1323(01)00034-8.

Comoglio, C., \& Botta, S. (2012). The use of indicators and the role of environmental management systems for environmental performances improvement: a survey on ISO 14001 certified companies in the automotive sector. Journal of Cleaner Production, 20(1), 92-102. http:// dx.doi.org/10.1016/j.jclepro.2011.08.022.

Diewert, W. E. (1976). Exact and superlative index numbers. Journal of Econometrics, 4(2), 115-145. http://dx.doi. org/10.1016/0304-4076(76)90009-9. 
Global Reporting Initiative - GRI. (2006). Sustainability reporting guidelines (Version 3.0). Amsterdam: GRI. Recuperado el 19 de noviembre de 2010, de https:// www.globalreporting.org/resourcelibrary/G3-Guidelines- Incl-Technical-Protocol.pdf.

Gunningham, N. (2009). Shaping corporate environmental performance: a review. Environmental Policy and Governance, 19(4), 215-231. http://dx.doi.org/10.1002/ eet.510.

Hernández, C. (2010). Modelo de gerenciamento da logística reversa integrado às questões estratégicas das organizações (Tesis doctoral). Universidade Estadual Paulista, Faculdade de Engenharia de Guaratinguetá, Guaratinguetá.

Hernández, C., Marins, F. A. S., Rocha, P. D., \& Duran, J. A. R. (2010). Using AHP and ANP to Evaluate the relation between reverse logistics and corporate performance in Brazilian Industry. Brazilian Journal of Operations \& Production Management, $7(2), 47-62$.

Hsu, C.-W., Hu, A. H., Chiou, C.-Y., \& Chen, T.-C. (2011). Using the FDM and ANP to construct a sustainability balanced scorecard for the semiconductor industry. Expert Systems with Applications, 38(10), 12891-12899. http://dx.doi.org/10.1016/j.eswa.2011.04.082.

Hubbard, G. (2009). Measuring organizational performance: beyond the triple bottom line. Business Strategy and the Environment, 18(3), 177-191. http://dx.doi.org/10.1002/ bse. 564 .

Ilinitch, A. Y., Soderstrom, N. S. \& Thomas, T. E. (1998). Measuring corporate environmental performance. Journal of Accounting and Public Policy, 17(4-5), 383-408.

Isaac, C. L., Díaz, S., La Rosa, M., Hernández, R., Hevia, F., \& Gómez, J. (2010). Indicadores para la evaluación del desempeño ambiental de los Centros de Educación Superior. Revista del Centro Nacional de Investigaciones Científicas, $41,1-12$.

ISO. (2015). ISO 14001 Sistemas de gestión ambiental — Requisitos con orientación para su uso. Suiza.

Lee, W.S., Huang, A. Y. H., Chang, Y.-Y., \& Cheng, C.M. (2011). Analysis of decision making factors for equity investment by DEMATEL and Analytic Network Process. 
Expert Systems with Applications, 38(7), 8375-8383. http://dx.doi.org/10.1016/j.eswa.2011.01.027.

Lober, D. (1996). Evaluating the environmental performance of corporations. Journal of Managerial Issues, 8(2), 184-205.

Luz, S. O. C., Sellitto, M. A., \& Gomes, L. P. (2006). Medição de desempenho ambiental baseada em método multicriterial de apoio à decisão: estudo de caso na indústria automotiva. Gestão \& Produção, 13(3), 557-570. http://dx.doi.org/10.1590/S0104$530 \times 2006000300016$.

Paoli, D., \& Moraes, L. A. D. F. (2011). Apoio multicritério à decisão como subsídio à gestão ambiental: o caso da Aggreko Brasil. Gestão \& Produção, 18(2), 379-390. http://dx.doi.org/10.1590/S0104-530X2011000200012.

Parra, V. L. R. (2005). Gestión de la información como herramienta para la evaluación del desempeño ambiental. Cub@: Medio Ambiente y Desarrollo, 5(9), 1-12.

Puolamaa, M., Kaplas, M., \& Reinikainen, T. (1996). Index of environmental friendliness: a methodological study. Helsinki: Statistics Finland.

Ramos, T., \& Melo, J. (2006). Developing and implementing an environmental performance index for the portuguese military. Business Strategy and the Environment, 15(2), 71-86. http://dx.doi.org/10.1002/bse.440.

Rao, P., Singh, A. K., O'Castillo, O., Intal, P. S., Jr., \& Sajid, A. (2009). A metric for corporate environmental indicators... for small and medium enterprises in the Philippines. Business Strategy and the Environment, 18(1), 14-31. http://dx.doi.org/10.1002/bse.555.

Raupp, F. (2012). Modelo de autoavaliação do desempenho ambiental para a agroindústria: o caso da indústria sucroalcooleira do Mato Grosso do Sul (Tesis doctoral). Universidade Federal de Santa Catarina, Florianópolis.

Saaty, R. W., \& Saaty, T. L. (2003). The Analytic Hierarchy Process (AHP) for decision making and the Analytic Network Process (ANP) for decision making with dependence and feedback. Pittsburgh: SuperDecisions. Recuperado el 13 de marzo de 2011, de http://www. superdecisions.com/demos_tutorials.php3.

Saaty, T. L. (1980). The analytic hierarchy process planning, priority setting, resource allocation. New York: McGraw-Hill. 
Saaty, T. L. (1996). Decision making with dependence and feedback: the analytic network process. Pittsburgh: RWS Publications.

Saaty, T. L. (2003). The seven pillars of the analytic hierarchy process. In T. L. Saaty \& L. G. Vargas (Eds.) Multiple criteria decision making in the new millennium (Cap. 2, pp. 23-40, Lecture Notes in Economics and Mathematical Systems, 507). Heidelberg: Springer- Verlag Berlin Heidelberg.

Sagastume, A., Van Caneghem, J., Cogollos, J. B., \& Vandecasteele, C. (2012). Evaluation of the environmental performance of lime production in Cuba. Journal of Cleaner Production, 31, 126-136. http://dx.doi.org/10.1016/j.jclepro.2012.02.035.

Sellitto, M., Borchardt, M., \& Medeiros, G. (2010). Modelagem para avaliação de desempenho ambiental em operações de manufatura. Gestão \& Produção, 17(1), 95-109.

Singh, K., Murty, H. R., Gupta, S. K., \& Dikshit, A. K. (2007). Development of composite sustainability performance index for steel industry. Ecological Indicators 7(3), 565588.https://www.researchgate.net/publication/222567616_Development_of_composit e_sustainability_performance_index_for_steel_industry 